\title{
Coefficient Estimates for Generalized Analytic Bi-Univalent Functions
}

Deborah Olufunmilayo Makinde

Department of Mathematics, Obafemi Awolowo University, Ile-Ife, Nigeria

\section{Abstract}

For the normalized analytic functions of the form

$$
f(z)=z+\sum_{n=2}^{\infty} a_{n} z^{n}, \quad a_{n} \in \mathbb{C},
$$

We obtain the initial coefficient estimates for the subclass $I_{\alpha \beta \gamma}^{S}(p, q ; \mu)$. The relationship with some coefficient estimates in the literature with that of the subclass above was also considered.

Keywords: Analytic; Bi-univalent; Linear transformation; Coefficient estimates.

(c) (i) CC BY: Creative Commons Attribution License 4.0

\section{Introduction}

Let $A$ be the class of normalized analytic functions $f$ in the open unit disc $U=\{z \in C:|z|<1\}$ with $f(0)=f^{\prime}(0)-1=0$ and of the form

$$
f(z)=z+\sum_{n=2}^{\infty} a_{n} z^{n}, \quad a_{n} \in \mathbb{C},
$$

and $S$ the class of all functions in $A$ that are univalent in $U$. This class of functions is defined on the entire complex plane, thus, one- to-one and onto. Hence, the inverse exists but the inverse may not be defined in the entire unit disc. It was established by Koebe in his one quarter theorem, that every univalent function maps the unit disc to a disc of radius $\frac{1}{4}$. The inverse function for every univalent function $S \in A$ can be defined by

and

$$
f^{-1}(f(z))=z,|z|<1
$$

$$
f^{-1}(f(w))=w, \quad\left(|w|<r_{0}(f) ; r_{0}(f) \geq \frac{1}{4}\right) \text {. }
$$

Let

$$
f(z)=\sum_{n=1}^{\infty} a_{n} z^{n}, \quad a_{1}=1,
$$

$\operatorname{And} f(z)=w$, using (3), we write

which is equivalent to

$$
f^{-1}(f(z))=\sum_{n=2}^{\infty} c_{n}(f(z))^{n}, \quad a_{1}=1
$$

$$
f^{-1}(w)=\sum_{n=1}^{\infty} c_{n} w^{n}, \quad a_{1}=1,
$$

equating coefficients in (2) and (4) with some calculations, we have

$$
f^{-1}(w)=w-a_{2} w^{2}+\left(2 a_{2}^{2}-a_{3}\right) w^{3}-\left(5 a_{2}^{3}-5 a_{2} a_{3}+a_{4}\right) w^{4}+\cdots
$$

Let $f^{-1}(w)=g(w)$, we write

$$
g(w)=w+\sum_{n=2}^{\infty} A_{n} w^{n}
$$

A function of the form (1) is said to be bi-univalent in $U$ if both $f$ and $f^{-1}=g$ are univalent in $U$. We denote the class of bi-univalent functions by $\Sigma$.

The study of the coefficient of bi-univalent functions began with the work of Jahangiri1 and Hamidi [1], Lewin [2], while the coefficient bounds of bi-univalent functions started with the work of Brannan and Taha [3]. This aspect of research has gained the attention of several researchers in the recent years and a lot of work has been done in this regard see [1, 4-8]. The author in Makinde, et al. [9] defined a certain linear operator by:

Definition: Let $s, \beta, \gamma \geq 0, \alpha$ a real number such that $s+\beta+\gamma>0$.

Then for a subclass $f$ of $A$, of the form:

$$
f(z)=z+\sum_{n=2}^{\infty} a_{n}^{i} z^{n}
$$


and $i\left(1 \leq i \leq k\right.$, the linear operator $I_{\alpha, \beta, \gamma}^{s} f$ is given by:

$$
\begin{gathered}
: I_{\alpha, \beta, \gamma} f(z)=\frac{\alpha f(z)+\beta z f^{\prime}(z)+\gamma z\left(z f^{\prime}(z)\right)^{\prime}}{\alpha+\beta+\gamma} \\
I_{\alpha, \beta, \gamma}^{2} f(z)=I_{\alpha, \beta, \gamma}\left(I_{\alpha, \beta, \gamma} f(z)\right) \\
I_{\alpha \beta \gamma}^{s} f(z)=I_{\alpha \beta \gamma}\left(I_{\alpha \beta \gamma}^{s-1} f(z)\right)
\end{gathered}
$$

and of the form

$$
I_{\alpha, \beta, \gamma}^{s} f(z)=z+\sum_{n=2}^{\infty}\left(\frac{\alpha+n \beta+n^{2} \gamma}{\alpha+\beta+\gamma}\right)^{s} a_{n}^{i} z^{n}
$$

The coefficients $a_{n}$ in (1) play a prominent role in analytic functions. The convergence of the analytic functions depend largely on $a_{n}$, for $|z|<1$. Thus, the importance of the coefficient estimates of the analytic functions cannot be overemphasized. Initial coefficient estimates is used in the study of Fekete-Szego functional and the Hankel determinant among others. Also, the author in Makinde [10] applied the generalized linear function introduced in Makinde, et al. [9] to a disease control measure using the coefficient estimate of the class of function. Motivated by the work of Mustafa [11], using the linear operator in (10) we define a new subclass of bi-univalent functions and obtained the initial coefficient estimates for the subclass. For the linear operator of the form (10), we obtain with simple calculations, the inverse function given by:

$$
\begin{aligned}
& I_{\alpha, \beta, \gamma}^{s} f^{-1}(w)=w-t_{2}^{s} a_{2} w^{2}+\left(t_{2}^{2 s} a_{2}^{2}-t_{3}^{s} a_{3}\right) w^{3}-\left(5 t_{2}^{3 s} a_{2}^{3}-5 t_{2}^{s} t_{3}^{s} a_{2} a_{3}+t_{4}^{s} a_{4}\right) w^{4} \\
& \begin{array}{c}
\text { Where } t_{2}=\left(\frac{\alpha+2 \beta+4 \gamma}{\alpha+\beta+\gamma}\right), t_{3}=\left(\frac{\alpha+3 \beta+9 \gamma}{\alpha+\beta+\gamma}\right), t_{4}=\left(\frac{\alpha+4 \beta+16 \gamma}{\alpha+\beta+\gamma}\right) \\
\text { Let } f^{-1}(w)=g(w), \text { we write } \\
g(w)=z+\sum_{n=2}^{\infty} A_{n} W^{n}
\end{array}
\end{aligned}
$$

In what follows, we define our subclass of analytic functions and obtain the estimates of the initial coefficients.

\section{Coefficient Estimates for the Subclass $\Sigma I_{\alpha, \beta, \gamma}^{S}(p, q ; \mu)$}

Definition 2 Let $p, q$ be analytic functions such that $p, q: U \rightarrow C, p(0)=q(0)=1$

and

$\min \{\operatorname{Re}(p(z)), \operatorname{Re}(p(z))\}>0, z \in U$.

Also, let $f$ be as in $(1)$, then $f$ is said to be in $\Sigma I_{\alpha, \beta, \gamma}^{s}(p, q ; \mu)$ if:

$f \in \Sigma$ and

$$
\begin{aligned}
& \left(I_{\alpha, \beta, \gamma}^{s} f(z)\right)^{\prime}+\mu z\left(I_{\alpha, \beta, \gamma}^{s} f(z)\right)^{\prime \prime} \in p(U), \quad z \in U, g \in \Sigma \\
& \text { and } \\
& \qquad\left(I_{\alpha, \beta, \gamma}^{s} g(w)\right)^{\prime}+\mu w\left(I_{\alpha, \beta, \gamma}^{s} g(w)\right)^{\prime \prime} \in p(U), w \in U \in p(U), w \in U,
\end{aligned}
$$

where $\mu>0$ and $\mathrm{I}_{\alpha, \beta, \gamma}^{S} f$ is as given in (10).

We now give the statement and the proof of the results in this study.

Theorem 1 Let $f \in \Sigma I_{(\alpha, \beta, \gamma)}^{S}(p, q ; \mu)$ where $\alpha, \beta, \gamma \geq 0, \alpha$ a real number

such that $\alpha, \beta, \gamma>0 ; s=0 ; 1 ; 2 ;::$ and $p ; q$ satisfy the condition in definition 1 . Then

and

$$
\left|a_{2}\right| \leq \min \left\{t_{2}^{-8} \frac{1}{2(1+\mu} \sqrt{\frac{\left|p^{\prime}(0)\right|^{2}+\left|q^{\prime}(0)\right|^{2}}{2}} \cdot t_{2}^{-8} \sqrt{\frac{\left|p^{\prime \prime}(0)\right|+\left|q^{\prime \prime}(0)\right|}{12(1+2 \mu)}}\right\}
$$

$$
\mid a_{3} \leq \min \left\{t_{3}^{-8} \frac{\left(\left|p^{\prime}(0)\right|^{2}\right.}{8(1+\mu)^{2}}+t_{3}^{-8} \frac{\left(\left|p^{\prime \prime}(0)\right|+\left|q^{\prime \prime}(0)\right|\right)}{12(1+2 \mu)}, t_{3}^{-8} \frac{\left|p^{\prime \prime}(0)\right|}{6(1+2 \mu)}\right\}
$$

Proof 1 Let $f \in \Sigma I_{\alpha, \beta, \gamma}^{s}(p, q ; \mu)$ and $I_{\alpha, \beta, \gamma}^{s} g=\left(I_{\alpha, \beta, \gamma}^{s} f\right)^{-1}$, thus by definition, we have respectively And

$$
\left(I_{\alpha, \beta, \gamma}^{s} f(z)\right)^{\prime}+\mu z\left(I_{\alpha, \beta, \gamma}^{s} f(z)\right)^{\prime \prime}=p(z), \quad z \in u
$$

$$
\left(I_{\alpha, \beta, \gamma}^{s} g(w)\right)^{\prime}+\mu w\left(I_{\alpha, \beta, \gamma}^{s} g(w)\right)^{\prime \prime}=q(w), \quad w \in u
$$

satisfies the condition of the definition 1 and

$$
\begin{aligned}
& p(z)=1+b_{1} z+b_{2} z^{2}+b_{3} z^{3}+\cdots \\
& q(w)=1+c_{1} w+c_{2} w^{2}+c_{3} w^{3}+\cdots
\end{aligned}
$$

Using (10) in (14) and (11) in (15) respectively give and

$$
1+2 t_{2}^{s} a_{2} z+3 t_{3}^{3} a_{3} z^{2}+\cdots+2 \mu t_{2}^{3} a_{2} z+\cdots 1+b_{1} z+b_{2} z^{2}+b_{3} z^{3}+\cdots
$$

$$
1-2 t_{2}^{s} a_{2} w+\left(6 t_{2}^{2 s} a_{2}^{2}-3 t_{3}^{3} a_{3}\right) w^{2}-\left(20 t_{2}^{3 s} a_{2}^{3}-20 a_{2} a_{3}+4 a_{4}\right) w^{3}+\cdots
$$




$$
\begin{aligned}
& \quad-2 \mu t_{2}^{s} a_{2} w+\mu\left(12 t_{2}^{2 s} a_{2}^{2}-6 t_{3}^{s} a_{3}\right) w^{2}-\mu\left(60 t_{2}^{3 s} a_{2}^{3}-60 a_{2} a_{3}+12 a_{4}\right) w^{3}+\cdots \\
& =1+c_{1} w+c_{2} w^{2}+c_{3} w^{3}+\cdots
\end{aligned}
$$

equating coefficients in (18) and (19), we obtain

$$
\begin{aligned}
& 2 t_{2}^{s}(1+\mu) a_{2}=b_{1} \\
& 3 t_{3}^{s}(1+2 \mu) a_{3}=b_{2}
\end{aligned}
$$

respectively

$-2 t_{2}^{s}(1+\mu) a_{2}=c_{1}$

$6 t_{2}^{2 s}(1+2 \mu) a_{2}^{2}-3 t_{3}^{s}\left((1+2 \mu) a_{3}=c_{2}\right.$

Comparing (20) and (22), we obtain

and

$$
c_{1}=-b_{1}
$$

$$
\begin{aligned}
& 4 t_{2}^{2 s}(1+\mu)^{2} a_{2}^{2}=b_{1}^{2} \\
& 4 t_{2}^{2 s}(1+\mu)^{2} a_{2}^{2}=c_{1}^{2}
\end{aligned}
$$

From (24) and (25), we have

Also, from (21) and (23), we have

$$
6 t_{2}^{2 s}\left((1+2 \mu)^{2} a_{2}^{2}=b_{2}+c_{2}\right.
$$

Now from (26) and (27), we have

$$
a_{2}^{2}=t_{2}^{-s} \frac{\left(b_{1}^{2}+c_{1}^{2}\right)}{8(1+\mu)^{2}}
$$

Respectively,

$$
a_{2}^{2}=t_{2}^{-2 s} \frac{\left(b_{2}+c_{2}\right)}{6(1+2 \mu)}
$$

Thus, from (28) and (29), using (16) and (17) we have

$$
\left|a_{2}\right|^{2} \leq t_{2}^{-2 s} \frac{\left(\left|p^{\prime}(0)\right|^{2}+\left|q^{\prime}(0)\right|^{2}\right.}{8(1+\mu)^{2}}
$$

Respectively,

$$
\left|a_{2}\right|^{2} \leq t 2^{-2 s} \frac{\left(\left|p^{\prime \prime}(0)\right|+\left|q^{\prime \prime}(0)\right|\right)}{12(1+2 \mu)}
$$

And from (30) and (31), we obtain our desired result for the coefficient $\left|a_{2}\right|$.

Furthermore, from (21) and (23), we have

$$
-6 t_{2}^{-2 s}(1+2 \mu) a_{2}^{2}+6_{3}^{t-3}(1+2 \mu) a_{3}=b_{2}-c_{2}
$$

Using (28) and (29) in (32), we obtain

$$
\left|a_{3}\right|=t_{3}^{-3} \frac{\left(b_{2}-c_{2}\right)}{6(1+2 \mu)}+t_{3}^{-3} \frac{\left(b_{1}^{2}-c_{1}^{2}\right)}{8(1+\mu)^{2}}
$$

And

$$
\left|a_{3}\right|=t_{3}^{-s} \frac{b_{2}}{3(1+2 \mu)}
$$

And from (33) and (34), we obtain our desired result for the

Coefficient $\left|a_{3}\right|$

Hence, we conclude the proof of the Theorem 1 .

\section{Remark 1}

1. It is noted that the generalize multiplier transform in Makinde, et al. [9] is an extension of Swammy's Swamy [12]. Thus, Theorem 2.1 will give the result for Swammy linear operator in place of the generalized multiplier transform by the author in Makinde, et al. [9] when $\gamma=0$

2. Also, when $\alpha=1 . \gamma=0$, the result for $N_{\beta}^{n}$ Swamy [12] is obtained in the Theorem. This is given in some of the corollaries below.

Corollary 1 Let $f \in I_{\alpha, \beta, \gamma}^{s}(p, q ; 1)$ where $s, \beta, \gamma \geq 0, \alpha$ a real number such that $\alpha+\beta+\gamma>0 ; s=0,1,2, \ldots$ and $p ; q$ satisfy the condition in definition 1. Then

And

$$
\left|a_{2}\right| \leq \min \left\{t_{2}^{-s} \frac{1}{4} \sqrt{\frac{\left|p^{\prime}(0)\right|^{2}+\left|q^{\prime}(0)\right|^{2}}{2}}, 6 t_{2}^{-s} \sqrt{\left|p^{\prime \prime}(0)\right|+\left|q^{\prime \prime}(0)\right|}\right\},
$$

$$
\left|a_{3}\right| \leq \min \left\{t_{3}^{-s} \frac{\left(|p \prime(0)|^{2}+\left|q^{\prime}(0)\right|^{2}\right)}{32}+t_{3}^{-s} \frac{\left(\left|p^{\prime \prime}(0)\right|+\left|q^{\prime \prime}(0)\right|\right)}{36}, t_{3}^{-s} \frac{|p \prime(0)|}{18}\right\} .
$$


Corollary 2 Let $f \in I_{\alpha, \beta, 0}^{s}(p, q ; \mu)$ where $s, \beta, \gamma \geq 0, \alpha$ a real number $\alpha+\beta>0 ; s=0,1,2, \ldots$ and $p ; q$ such that satisfy the condition in definition 1. Then

and

$$
\left|a_{2}\right| \leq \min \left\{t_{2 \alpha \beta}^{-s} \frac{1}{2(1+\mu)} \sqrt{\frac{\left|p^{\prime}(0)\right|^{2}+\left|q^{\prime}(0)\right|^{2}}{2}, t_{2 \alpha \beta}^{-s} \sqrt{\frac{\left|p^{\prime \prime}(0)\right|+\left|q^{\prime \prime}(0)\right|}{12(1+\mu)}}}\right\},
$$

where

$$
\left|a_{3}\right| \leq \min \left\{t_{3 \alpha \beta}^{-s} \frac{\left|p^{\prime}(0)\right|^{2}+\left|q^{\prime}(0)\right|^{2}}{8(1+\mu)^{2}}+t_{3 \alpha \beta}^{-s} \frac{\left|p^{\prime \prime}(0)\right|+\left|q^{\prime \prime}(0)\right|}{12(1+2 \mu)}, t_{3 \alpha \beta}^{-s} \frac{\left|p^{\prime \prime}(0)\right|}{6(1+2 \mu)}\right\}
$$

$t_{3 \alpha \beta}=\frac{\alpha+\beta}{\alpha+2 \beta}$ and $t_{3 \alpha \beta}=\frac{\alpha+\beta}{\alpha+3 \beta}$

Remark 2 The corollary 2 gives the result for the Swammy's linear operator for the subclass of this study.

Corollary 3 Let $f \in I_{\alpha, \beta, 0}^{s}(p, q ; 1)$ where $s, \beta, \gamma \geq 0, \alpha$ a real number $\alpha+\beta>0 ; s=0,1,2, \ldots$ and $p$; $q$ such that satisfy the condition in definition 1 . Then

and

$$
\left|a_{2}\right| \leq \min \left\{t_{2 \alpha \beta}^{-s} \frac{1}{4} \sqrt{\frac{\left|p_{\prime}^{\prime}(0)\right|^{2}+\left|q^{\prime}(0)\right|^{2}}{2}}, 6 t_{2 \alpha \beta}^{-s} \sqrt{\left|p^{\prime \prime}(0)\right|+\left|q^{\prime \prime}(0)\right|}\right\}
$$

$$
\left|a_{3}\right| \leq \min \left\{t_{3 \alpha \beta}^{-s} \frac{\left|p^{\prime}(0)\right|^{2}+\left|q^{\prime}(0)\right|^{2}}{32}+t_{3 \alpha \beta}^{-s} \frac{\left|p^{\prime \prime}(0)\right|+\left|q^{\prime \prime}(0)\right|}{36}, t_{3 \alpha \beta}^{-s} \frac{\left|p^{\prime \prime}(0)\right|}{18}\right\}
$$

where

$$
t_{3 \alpha \beta}=\frac{\alpha+\beta}{\alpha+2 \beta} \text { and } t_{3 \alpha \beta}=\frac{\alpha+\beta}{\alpha+3 \beta}
$$

Corollary 4 Let $f \in I_{1, \beta, 0}^{s}(p, q ; \mu)$ where $\beta \geq 0 ; s-0,1,2 \ldots$ and $p, q$ satisfy the condition in definition 1 . Then

And

$$
\left|a^{2}\right| \leq \min \left\{t_{2 \beta}^{-s} \frac{1}{2(1+\mu} \sqrt{\frac{\left|p^{\prime}(0)\right|^{2}+\left|q^{\prime}(0)\right|^{2}}{2}}, t_{2 \beta}^{-s} \sqrt{\frac{\left|p^{\prime \prime}(0)\right|+\left|q^{\prime \prime}(0)\right|}{12(1+2 \mu)}}\right\},
$$

$$
\left|a_{3}\right| \leq \min \left\{t_{3 \beta}^{-s} \frac{\left|p^{\prime}(0)\right|^{2}+\left|q^{\prime}(0)\right|^{2}}{8(1+\mu)^{2}}+t_{3 \beta}^{-s} \frac{\left|p^{\prime \prime}(0)\right|+\left|q^{\prime \prime}(0)\right|}{12(1+2 \mu)}, t_{3 \text { beta }}^{-s} \frac{\left|p^{\prime \prime}(0)\right|}{6(1+2 \mu)}\right\} .
$$

Remark 3 The corollary 2 gives the result the operator $N_{\beta}^{n}(z)$ for the subclass of this study.

Corollary 5 Let $f \in I_{1, \beta, 0}^{s}(p, q ; 1)$ where $\beta \geq 0, s=0,1,2, \ldots$ and $p, q$

satisfy the condition in definition 1. Then

and

$$
\left|a_{2}\right| \leq \min \left\{t_{2 \beta}^{-s} \frac{1}{4} \sqrt{\frac{|p \prime(0)|^{2}+\left|q^{\prime}(0)\right|^{2}}{2}}, 6 t_{2 \beta}^{-8} \sqrt{\left|p^{\prime}(0)\right|+\left|q^{\prime}(0)\right|}\right\},
$$

$$
\left|a_{3}\right| \leq \min \left\{t_{3 \beta}^{-s} \sqrt{\frac{\left|p^{\prime}(0)\right|^{2}+\left|q^{\prime}(0)\right|^{2}}{32}}+t_{3 \beta}^{-s} \frac{\left(\left|p^{\prime}(0)\right|+\left|q^{\prime}(0)\right|\right)}{36}, t_{3 \beta}^{-s} \frac{\left|p^{\prime}(0)\right|}{18}\right\}
$$

\section{Conclusion}

The generalized multiplier transform in Makinde, et al. [9] which is an extension of the Swammy's linear operator in Swamy [12] was applied to the method of Mustafa in Mustafa [11]. The results in this study extends that of its form in the literature.

\section{References}

[1] Jahangiri1, J. M. and Hamidi, S. G., 2013. "Coefficient estimates for certain classes of bi-univalent functions." International Journal of Mathematics and Mathematical Sciences, vol. 2013, pp. 1-4.

[2] Lewin, M., 1967. "On a coefficient problem for bi-univalent functions." Proceedings of the American Mathematical Society, vol. 18, pp. 63-68.

[3] Brannan, D. A. and Taha, T. S., 1986. "On some classes of bi- univalent functions, studia universitatis babes-bolyai." Mathematica, vol. 31, pp. 70-77.

[4] Ali, R. M., Lee, S. K., Ravichandran, V., and Supramaniam, S., 2012. "Coefficient estimates for biunivalent ma-minda starlike and convex functions." Applied Mathematics Letters, vol. 25, p. $344 ? 351$.

[5] Frasin, B. A. and Aouf, M. K., 2011. "New subclasses of bi- univalent functions." AppliedMathematics Letters, vol. 24, pp. 1569-1573.

[6] Hamidi, S. G., Halim, S. A., and Jahangiri, J. M., 2013. "Faber polynomial coefficient estimates for meromorphic bi-starlike functions." International Journal of Mathematics and Mathematical Sciences, vol. 2013, pp. 1-4. 
[7] Srivastava, H. M., Mishra, A. K., and Gochhayat, P., 2010. "Certain subclasses of analytic and bi-univalent functions?" Applied Mathematics Letters, vol. 23, pp. 1188-1192.

[8] Vijaya, R., Sudharsan, T. V., and Sivasubramanian, S., 2016. "Coefficient estimates for certain Subclasses of biunivalent functions defined by convolution." International Journal of Analysis, vol. 2016, pp. 1-5.

[9] Makinde, D. O., Hamzat, J. O., and Gbolagade, A. M., 2019. "A generalized multiplier transform on a univalent integral operator." Journal of Contemporary Applied Mathematics, vol. 9, pp. 31-38.

[10] Makinde, D. O., 2019. "A generalized multiplier transform on a p-valent integral operator with application." American Journal of Applied Mathematics and Statistics, vol. 7, pp. 115-119.

[11] Mustafa, N. "The various properties of certain subclasses of analytic functions of complex order." pp. 1-9. Available: https://arxiv.org/ftp/arxiv/papers/1704/1704.04980.pdf

[12] Swamy, S. R., 2012. "Inclusion Properties of certain subclasses of analytic functions." International Mathematical Forum, vol. 7, pp. 1751-1760. 